\title{
Prévision des concentrations maximales quotidiennes d'ozone en Bourgogne en 2003
}

Daily maximum ozone forecast for 2003 in Burgundy

Tägliche Vorhersage der maximalen Ozonkonzentration für 2003 im Burgund

Yves Richard, Marie-Laure Houze et Serge Taboulot

\section{OpenEdition}

\section{Journals}

Édition électronique

URL : http://journals.openedition.org/rge/1369

DOI : $10.4000 /$ rge.1369

ISSN : 2108-6478

Éditeur

Association des géographes de l'Est

Édition imprimée

Date de publication : 1 janvier 2006

ISSN : 0035-3213

\section{Référence électronique}

Yves Richard, Marie-Laure Houze et Serge Taboulot, « Prévision des concentrations maximales quotidiennes d'ozone en Bourgogne en 2003 », Revue Géographique de l'Est [En ligne], vol. 46 / 1-2 2006, mis en ligne le 18 décembre 2009, consulté le 08 septembre 2020. URL : http:// journals.openedition.org/rge/1369; DOI : https://doi.org/10.4000/rge.1369

Ce document a été généré automatiquement le 8 septembre 2020.

Tous droits réservés 


\title{
Prévision des concentrations maximales quotidiennes d'ozone en Bourgogne en 2003
}

\author{
Daily maximum ozone forecast for 2003 in Burgundy \\ Tägliche Vorhersage der maximalen Ozonkonzentration für 2003 im Burgund
}

Yves Richard, Marie-Laure Houze et Serge Taboulot

\section{La prévision de l'ozone : ses enjeux}

1 En Bourgogne, durant la période estivale, comme dans la plupart des régions françaises, les indices de qualité de l'air élaborés et communiqués par les AASQA (Associations Agréées de Surveillance de la Qualité de l'Air) sont majoritairement influencés par les concentrations atmosphériques en ozone. En effet ce polluant estival, à l'exception des abords des zones industrielles, est celui qui a le plus d'effet sur la santé humaine (Mégie, 1993). Ainsi, la prévision des concentrations en ozone est devenue un enjeu important en terme de santé publique.

2 Pour effectuer ces prévisions, il faut prendre en compte le fait que l'ozone est un polluant secondaire issu de processus complexes. Interviennent dans sa formation des précurseurs (oxydes d'azote (Nox) et Composés Organiques Volatils Non Méthaniques (COVNM)) transformés lors de conditions météorologiques favorables (température et ensoleillement élevés, vents faibles). Ainsi, en Scandinavie, la diminution des émissions de précurseurs amorcée depuis 1990 commence à avoir des effets positifs sur la pollution à l'ozone (Solberg et al., 2005).

Une collaboration entre l'université de Bourgogne, Météo-France et ATMOSF'air a été initiée à cette intention. L'année 2003 a servi de période test pour douze stations situées dans quatre agglomérations (Dijon, Chalon-sur-Saône, Macon et Montceau-lesMines) ainsi qu'à Saint-Brisson, station rurale du massif du Morvan (Figure 1). L'approche par station permet de confronter prévisions et observations et de prendre 
en compte les différences importantes observées quant aux concentrations d'ozone entre centres et périphéries des villes (Bavay et Roussel, 1992 ; Richard et al., 2000 ; Jimenez et al., 2005). La période se limite à la saison chaude et ensoleillée ( $1^{\mathrm{er}}$ avril - 30 septembre) pour laquelle les concentrations d'ozone sont maximales, en particulier quand prédominent des conditions anticycloniques (Bavay et Roussel, 1992 ; Mégie et al., 1993 ; Richard et al., 2000).

Figure 1 : Stations ATMOSF'air, Météo France et mailles ALADIN.

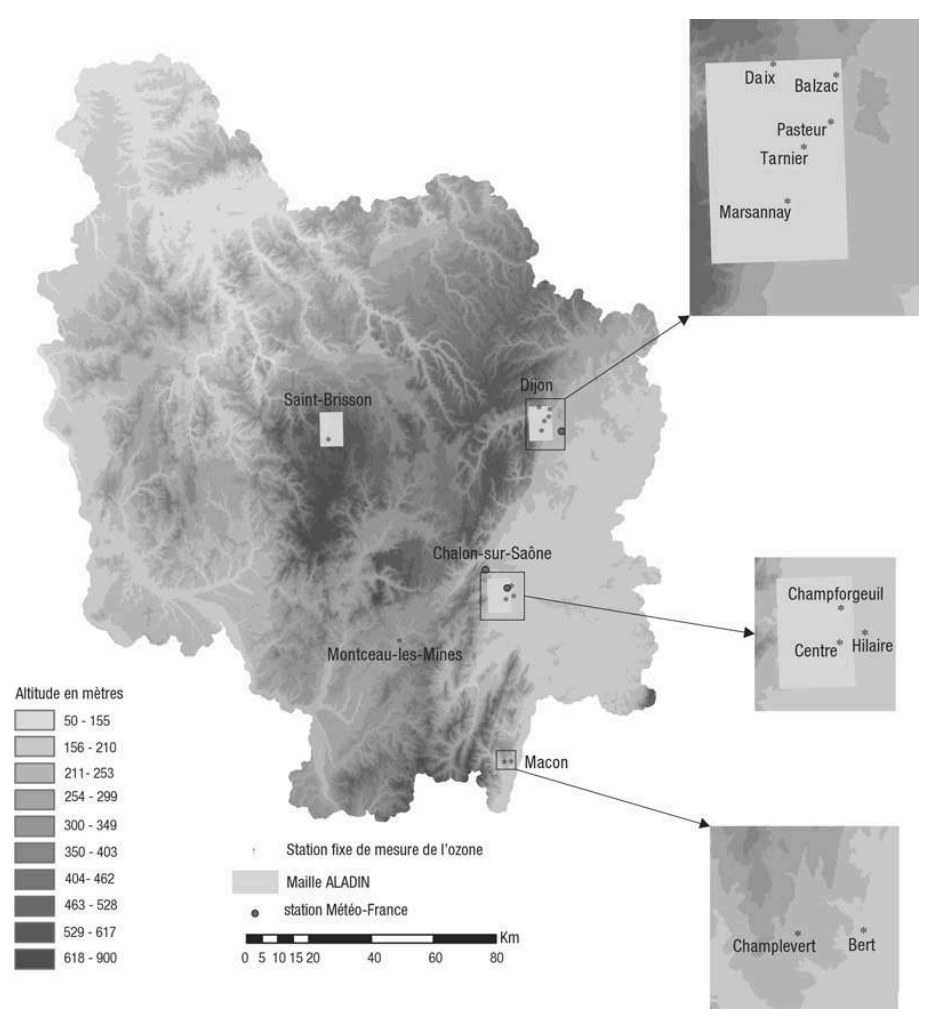

\section{Une méthode statistico-dynamique}

Les différentes prévisions de l'ozone peuvent être effectuées selon deux familles de méthodes :

5 1) purement numérique : les prévisions sont issues de modèles de prévisions météorologiques comprenant un module chimie-transport. Ces approches sont développées dans plusieurs régions françaises, par exemple pour l'île-de-France (Vautard et al., 2000) ou la Provence (Durand et Cros, 2004);

6 2) statistico-dynamique : des outils statistiques sont développés en aval de modèles numériques (en l'occurrence des modèles de prévisions météorologiques) intégrant ou non la chimie. La composante statistique de ces modèles s'appuie sur des régressions multiples, des analyses discriminantes, des réseaux de neurones, des méthodes de recherche de cas passés similaires ou des arbres de décisions (Fromage et Gilibert 1997 ; Ghiaus, 2005 ; McMillan et al., 2005).

7 La méthode statistico-dynamique, connue et testée en météorologie opérationnelle sous la dénomination d'adaptations statistiques de sortie de modèle, est la base retenue pour cette étude de performance des prévisions locales de concentrations d'ozone. 


\section{Données}

\section{A. Le prédictand : L'ozone troposphérique}

8 Le prédictand est la valeur à prévoir: la concentration maximale d'ozone du lendemain $(J+1)$. Pour chaque jour, nous avons sélectionné la valeur maximale d'ozone entre 9 $\mathrm{h}$ et $14 \mathrm{~h}$ UTC, tranche horaire où se situent généralement les valeurs les plus élevées (Houzé, 1999). Ce rythme diurne des concentrations en ozone est associé à l'insolation mais aussi à l'épaisseur de la couche limite (Bavay et Roussel, 1992). En outre, ce choix horaire permet une prévision à partir de $16 \mathrm{~h}$ légales en été (14 h UTC), horaire compatible avec le souci opérationnel de pouvoir déclencher, le cas éventuel, les procédures d'information des autorités et de la population.

\section{B. Les prédicteurs chimiques ou précurseurs}

9 Nous ne présenterons ici que les prédicteurs chimiques mesurés par ATMOSF'air ${ }^{1}$ dans les 12 stations (Figure 1) et sélectionnés par les modèles :

10 1) l'ozone du jour (J) permet de prendre en compte un éventuel phénomène d'accumulation (Vergez, 1999);

11 2) les oxydes d'azote (NOx) constituent l'un des principaux précurseurs de la pollution photochimique (Jacob, 2000).

12 Pour chacun, deux valeurs sont considérées:

13 1) la moyenne $9 \mathrm{~h}-14 \mathrm{~h}$ UTC du jour J, qui minimise l'impact des variations rapides (haute fréquence) ;

14 2) le maximum $9 \mathrm{~h}-14 \mathrm{~h}$ UTC du jour J, étant donné qu'un changement de temps rapide en cours de journée peut parfois rendre la moyenne peu pertinente.

\section{Les prédicteurs météorologiques}

15 Ils sont tous issus ou dérivés d'ALADIN, version à domaine limité du modèle ARPEGE de Météo-France. ALADIN-France permet de gagner un facteur 2 par rapport à la résolution spatiale la plus forte d'ARPEGE auquel il est couplé (Pailleux et al., 2000). La version utilisée est donc la base opérationnelle des prévisions atmosphériques, lancée chaque nuit à 00h UTC. Les simulations sont archivées selon des échéances séparées de $3 \mathrm{~h}$. Nous en avons retenues deux :27 et 36 heures qui permettent de documenter : 1 ) la fin de nuit (échéance $27,03 \mathrm{~h}$ UTC., soit $5 \mathrm{~h}$ légales en été, moment où les températures comme les concentrations d'ozone sont minimales) ;2) le début d'après midi (échéance 36, 12 h UTC., soit 14 h légales, moment de la journée ou températures et ozone atteignent leur valeurs maximales).

Nous utilisons, pour trois points de grille centrés sur Dijon, Chalon-sur-Saône et SaintBrisson (Figure 1), des valeurs prévues au niveau du sol (température, pression, flux solaires et thermiques, précipitations liquides de convection et précipitations liquides à grande échelle) ainsi que sur 11 niveaux $(2,10,20,50,100,250,500,750,1$ 000, 1 250, 1 $500 \mathrm{~m}$ ) au-dessus du sol (température, composantes horizontales et verticales du vent, 
humidité relative). Enfin, nous intégrons la nébulosité du sol à $1500 \mathrm{~m}$, de $1500 \mathrm{~m}$ à $5 \mathrm{~km}$, au-delà de $5 \mathrm{~km}$ ainsi que totale.

17 À partir de ces variables, nous en avons calculé l'amplitude diurne, ou encore la différence entre deux niveaux d'altitude afin de prendre en compte la stratification verticale dans les basses couches. L'ensemble représente au total 170 prédicteurs. A noter des valeurs manquantes dans les données qui interdisent les prévisions (cas de la fin juin 2003 par exemple). Pour Dijon et Chalon-sur-Saône, la pertinence des prévisions météorologiques est validée par les valeurs réellement observées aux stations de Dijon-Longvic pour le point de grille " Dijon », Chalon-Champforgeuil et Rully (rayonnement global) pour le point de grille « Chalon » (Figure 1).

\section{Méthode}

\section{A. Régression linéaire multiple pas à pas}

La régression linéaire multiple consiste à expliquer un phénomène (ici les concentrations maximales d'ozone à $\mathrm{J}+1$ ), par la conjonction des variations de plusieurs phénomènes. Les variables sont sélectionnées pas à pas (stepwise). Il s'agit d'un algorithme de régression utilisant les coefficients de corrélation partielle.

\section{B. Période d'apprentissage : 1999-2002}

Les équations statistiques utiles à la prévision doivent être construites avec des données utilisées pour caler les paramètres qui diffèrent des données servant à évaluer les résultats. On sépare les observations en jeu d'apprentissage d'une part (1999-2002) et jeu de validation d'autre part (2003). Sur la période d'apprentissage, nous avons utilisé la procédure de test dite Jack-knife, s'apparentant à la validation croisée dans la mesure où l'on retire tour à tour chaque observation de l'échantillon d'apprentissage. Ceci permet une validation à l'aide de données différentes de celles qui ont servi à l'ajustement de la droite. Cette méthode permet d'évaluer la performance réelle d'un modèle de prévision avant qu'il ne soit appliqué (Thiria et al., 1997). Nous obtenons, sur la période d'apprentissage (1999-2002), et ce pour chaque station, une équation de régression sélectionnant un maximum de 5 prédicteurs parmi les 170 soumis, sachant que ces 5 prédicteurs peuvent varier selon la station. Ce nombre maximal imposé privilégie le dynamisme des modèles sur leur performance en termes de corrélations.

Les séries de prévisions ainsi obtenues ont des variances trop faibles, ce pour l'ensemble des stations ATMOSF'air. Pour pallier ce problème, classique en adaptation statistique de sortie de modèle météorologique, le correctif K (Ward et Folland, 1991) renforce le " dynamisme » des prévisions car augmente l'écart type de la prévision en l'ajustant à celui de l'observation de la période d'apprentissage (1999-2002).

\section{Période de validation : 2003 , un été d'exception}

21 À partir des températures horaires relevées dans les stations de Météo-France (DijonLongvic ou Chalon-Champforgeuil) nous avons calculé la " climatologie " de la période d'apprentissage en effectuant une moyenne sur les 4 ans (Figure 2a). Celle-ci se caractérise par l'addition du cycle diurne (en ordonnées) et du régime saisonnier (en 
abscisses) sur lesquels se surimposent des fluctuations météorologiques indépendantes du calendrier et de l'heure. Ces fluctuations ne sont pas totalement lissées en raison de la brièveté de l'échantillon (4 ans). Par exemple, le hasard a fait, qu'à ChalonChampforgeuil, la première décade d'août fut plutôt fraîche lors des étés 1999-2002 (Figure 2a).

Les températures relevées sur la période de test (Figure $2 b$ ) présentent les mêmes caractères avec toutefois :

1) des fluctuations horaires et surtout d'un jour à l'autre plus marquées (il ne s'agit pas d'une moyenne) ;

2) des températures globalement bien plus élevées, caractéristiques du printemps et de l'été 2003 sur une bonne part de l'Europe de l'ouest (Grazzini et al., 2003).

La période dite " de canicule " s'individualise bien avec des températures dépassant chaque jour $35{ }^{\circ} \mathrm{C}$ entre les 3 et 13 août, $38{ }^{\circ} \mathrm{C}$ entre les 5 et 13 août (excepté le 8) et culminant à $39,2{ }^{\circ} \mathrm{C}$ le 12 août (Figure $2 b$ ).

Figure 2 : Températures horaires en avril-septembre à Châlon-Champforgeuol (en ${ }^{\circ} \mathrm{C}$ ).

a : période d'apprentissage : moyenne 1999-2002.

$b$ : période de test : 2003 .
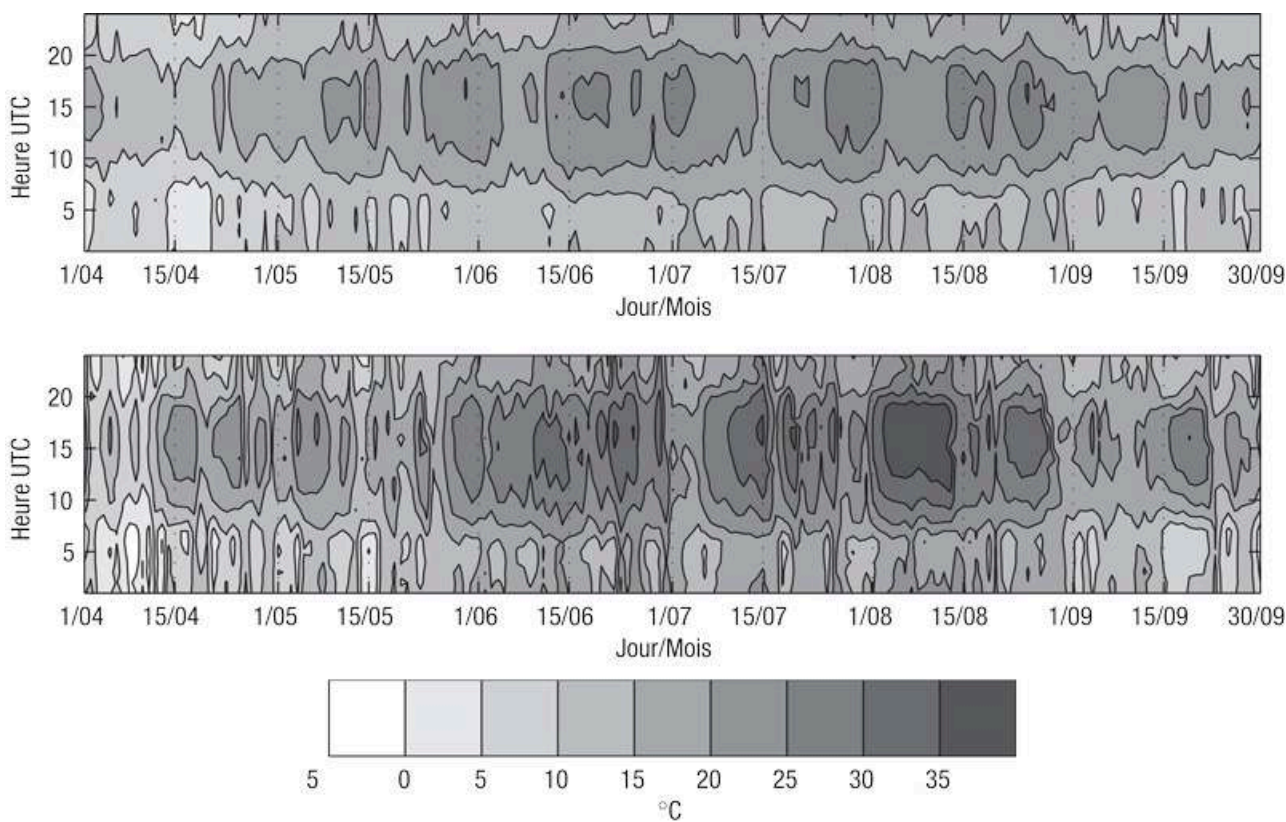

Les mêmes calculs sont effectués sur les valeurs d'ozone relevées dans 12 stations ATMOSF'air. Nous présentons ceux de Chalon-Champforgeuil, station située à proximité d'une station météorologique. Sur la période d'apprentissage (Figure 3a), les concentrations moyennes d'ozone sont généralement inférieures à $40 \mu \mathrm{g} / \mathrm{m}^{3}$ la nuit. Elles sont comprises entre 80 et $120 \mu \mathrm{g} / \mathrm{m}^{3}$ lors des après-midi d'été. Ces valeurs relativement faibles signifient que la pollution est globalement faible. Ceci n'exclue pas des épisodes marqués qui, n'intervenant pas les mêmes jours d'une année à l'autre, n'apparaissent pas de matière nette sur une moyenne. Cette forte variabilité inter annuelle explique que sur un échantillon court (4 ans) les fluctuations soient fortes 
d'un jour à l'autre. Ainsi n'y a-t-il par exemple pas d'autre interprétation que la brièveté de la période d'apprentissage pour expliquer les faibles concentrations observées vers le 15 juillet (Figure 3a).

Le printemps et l'été 2003 (Figure $3 b$ ) se caractérisent par la présence de plusieurs séquences où les concentrations en ozone sont élevées (fin avril, première quinzaine de juin, mi-juillet, première quinzaine d'août, ...). Ces épisodes d'ozone sont à mettre en relation avec les conditions météorologiques exceptionnelles (fort ensoleillement et température élevée). Ainsi, lors de la canicule (dimanche 3 au mercredi 13 août), la valeur seuil légale d'information et de recommandation de $180 \mu \mathrm{g} / \mathrm{m}^{3}$ a été dépassée lors de 6 journées à la station de Chalon-Champforgeuil (6, 7, 9, 10, 12 et 13 août).

Figure 3 : Concentrations horaires en avril-septembre à Chalon-Champforgeuil (en $\mu \mathrm{g} / \mathrm{m}^{3}$ ).

a : période d'apprentissage : moyenne 1999-2002.

$b$ : période de test: 2003.
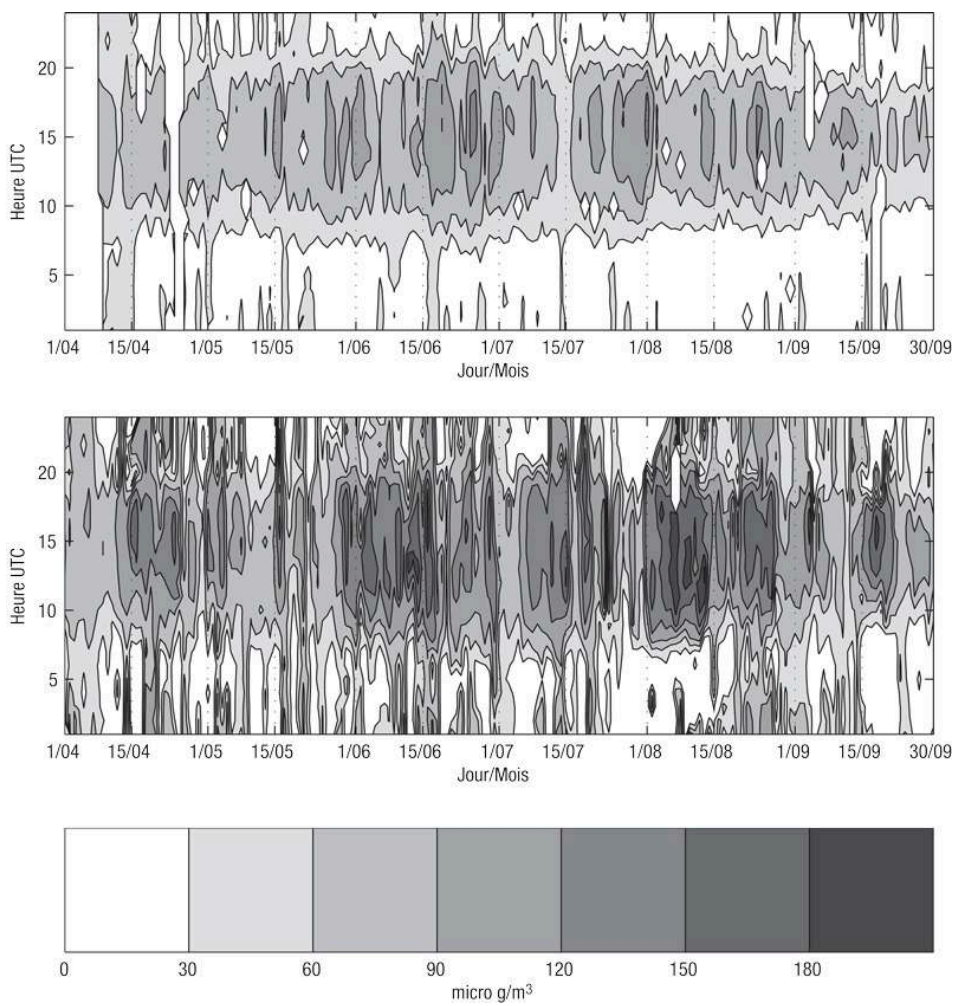

Du point de vue du prévisionniste, ces conditions, tant d'ozone que météorologiques, signifient que la période de test comporte des valeurs inconnues en période d'apprentissage. L'enjeu est bien de savoir si, dans de telles conditions, les modèles statistico-dynamiques, à l'image des modèles dynamiques (Vautard et al., 2005) sont susceptibles de fournir des prévisions réalistes, gage de leur robustesse. 


\section{Résultats}

\section{A. Des prévisions réalistes} compte les conditions de formation de l'ozone. Les coefficients affectés à ces trois types de prédicteurs sont positifs. Aux autres prédicteurs (humidité, vent, température nocturne) sont associés des coefficients négatifs. Les phénomènes de destruction, de dépôt et de dispersion de l'ozone sont ainsi également pris en compte, mais potentiellement de manière un peu moins efficace

Nous détaillons trois équations dédiées à des stations " périurbaines " de Saône-etLoire :

Chalon-Champforgeuil: $\mathrm{O}_{3 \max }$ Pré $=44.5+\left(0.46 \mathrm{O}_{3 \max }\right)+\left(4.41 \mathrm{~T}_{1}\right)-\left(2.84 \mathrm{t}_{1}\right)-\left(0.36 \mathrm{H}_{11}\right)+$ $\left(1.9110^{-6} \mathrm{~F}_{-} \mathrm{Th}\right)$

Macon-Champlevert: $\mathrm{O}_{3 \max }$ Pré $=55.7+\left(0.44 \mathrm{O}_{3 \text { mox }}\right)+\left(4.49 \mathrm{~T}_{1}\right)-\left(2.72 \mathrm{t}_{1}\right)-\left(0.37 \mathrm{H}_{11}\right)+(1.9$ $\left.10^{-6} \mathrm{~F} \_\mathrm{Th}\right)$

Montceau-Ecluse: $\mathrm{O}_{3 \max }$ Pré $=73.6+\left(0.52 \mathrm{O}_{3 \max }\right)+\left(1.28 \mathrm{~T}_{1}\right)-\left(0.45 \mathrm{H}_{8}\right)-(1.07 \mathrm{U} 10)+(1.91$ $\left.10^{-6} \mathrm{~F} \_\mathrm{Th}\right)$

Avec:

$\mathrm{O}_{3 \max }$ Pré : concentration maximale d'ozone prévue pour le lendemain $(\mathrm{J}+1)$

$\mathrm{O}_{3 \max }$ concentration maximale mesurée à la station par ATMOSF'air le jour J entre $9 \mathrm{~h}$ et $14 \mathrm{~h}$ UTC

$\mathrm{O}_{3 \text { moy: }}$ concentration moyenne mesurée à la station par ATMOSF'air le jour J entre $9 \mathrm{~h}$ et $14 \mathrm{~h} \mathrm{UTC}$

$\mathrm{T}_{1}$ : température ALADIN échéance $36 \mathrm{~h}$, soit J $+112 \mathrm{~h} \mathrm{UTC}$, à $2 \mathrm{~m}$

$\mathrm{t}_{1:}$ température ;ALADIN échéance $27 \mathrm{~h}$, soit $\mathrm{J}+13 \mathrm{~h} \mathrm{UTC}$, à $2 \mathrm{~m}$

$\mathrm{H}_{11}$ : humidité ALADIN échéance 36h, soit J + 112 h UTC, à $1500 \mathrm{~m}$

$\mathrm{H}_{8}$ : humidité ALADIN échéance 36h, soit J + 112 h UTC, à $750 \mathrm{~m}$

F_Th: flux thermique ; ALADIN échéance $36 \mathrm{~h}$, soit J + $112 \mathrm{~h}$ UTC

U10 : composante zonale du vent ALADIN échéance $36 \mathrm{~h}$, soit J + $112 \mathrm{~h}$ UTC, à 1250 $\mathrm{m}$

Les prédicteurs sélectionnés varient peu d'une station à l'autre. Deux types de prédicteurs n'entrent que dans certains modèles : le vent et la température nocturne et quatre types de prédicteurs sont systématiquement pris en compte :

1) les concentrations en ozone du jour ;

2) la température à $2 \mathrm{~m}$ prévue pour le lendemain à $12 \mathrm{~h}$ UTC ;

3) l'humidité relative de l'air prévue à $12 \mathrm{~h}$ UTC (à différents niveaux d'altitude selon la station);

4) le flux thermique prévu à $12 \mathrm{~h}$ UTC.

La présence de l'ozone du jour dans les modèles traduit l'inertie des concentrations d'un jour à l'autre (auto corrélation de rang 1) tout particulièrement en phase d'accumulation. Celle de la température et du rayonnement à $12 \mathrm{~h}$ UTC. prend en Plusieurs indices statistiques donnent une mesure de la performance de ces modèles. Nous avons retenu le coefficient de détermination $\left(\mathrm{R}^{2}\right)$ qui indique la part commune de 
variance des concentrations maximales d'ozone observées et prévues (Figure 4). On retiendra que les performances sont globalement très bonnes (minimum de 0.63 pour Dijon-Pasteur et maximum de 0.78 pour les deux stations mâconnaises) et qu'elles diffèrent peu d'une station à l'autre.

Figure 4 : Coefficients de détermination des 12 modèles.

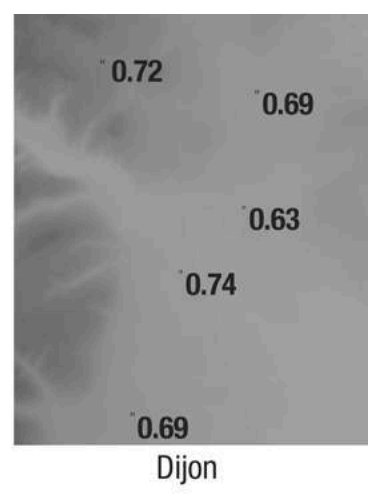

Saint-Brisson

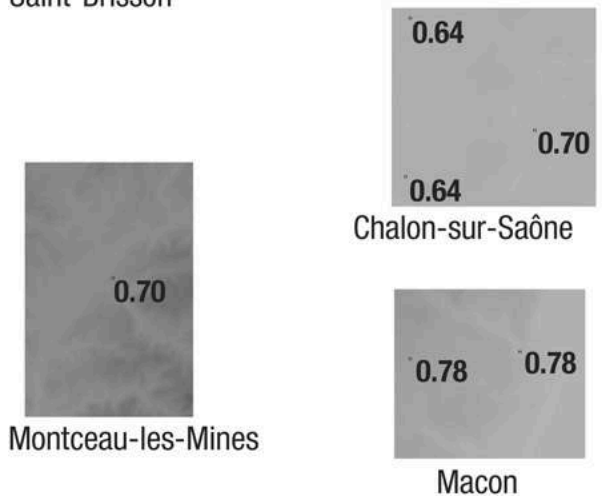

Pour Chalon-Champforgeuil, une des stations où le $\mathrm{R}^{2}$ est le moins élevé, les grandes phases (épisodes comme jours à faibles concentrations) sont globalement bien prévues (Figure 5). De surcroît les niveaux prévus de pollution sont conformes aux observations. Ce second point est fondamental et très encourageant car il montre l'aptitude de notre démarche à prévoir les valeurs extrêmes avec de bons ordres de grandeur : les pics de pollution en ozone sont donc prévisibles quantitativement par des méthodes statisticodynamiques, ce qui était loin d'être un résultat acquis au départ, la prévision des extrêmes étant toujours la plus délicate. 
Figure 5 : Concentrations quotidiennes maximales en ozone observées et prévues pour ChalonChampforgeuil (2 avril - 30 septembre 2003)

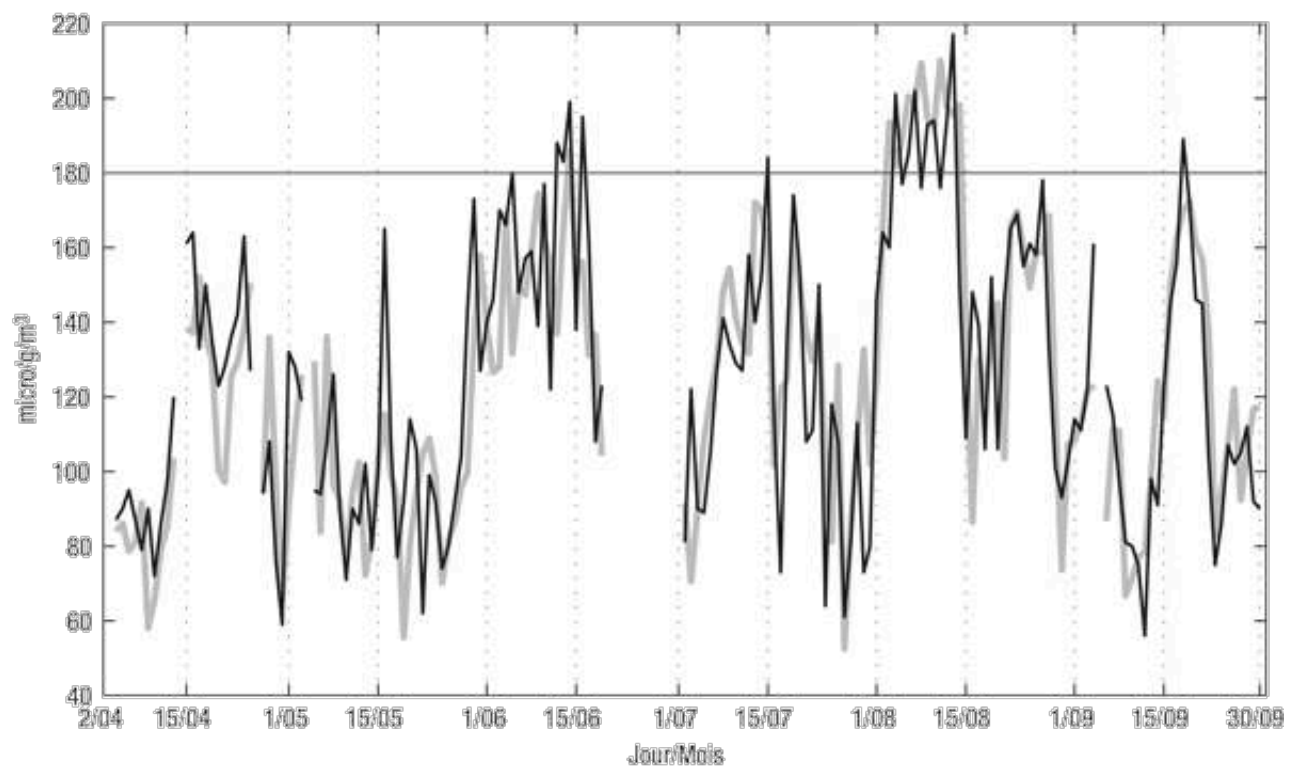

Noir fin: observé ; Grisé épais : prévu

\section{B. Diagnostics des principales erreurs de prévisions}

Sur les différentes stations l'erreur moyenne de prévision est de l'ordre de $20 \mu \mathrm{g} / \mathrm{m} 3$. Dans le détail, le recouvrement entre séries observée et prévue est imparfait. Un zoom effectué sur la canicule et les jours qui l'encadrent montre un décalage : les prévisions ont tendance à suivre les observations avec un jour de retard, c'est très net les $(1,3,4$, 8,11 et 14 août (Figure 6). Pour tester l'inertie induite par le prédicteur " ozone du jour ", nous avons calculé les corrélations entre les deux courbes (observé et prévu) avec un décalage d'un jour. Les résultats montrent que les corrélations entre l'ozone prévu à $\mathrm{J}+1$ et l'ozone observé sont de même ordre selon que l'on considère l'ozone du lendemain $(J+1)$ ou l'ozone du jour $(J)$. Cela signifie que le prédicteur « ozone du jour » joue un rôle déterminant dans la prévision et restreint la réactivité des modèles. Ce problème peut théoriquement être important dans les situations de changement de temps rapides (c'est le cas du 14 août), malgré l'introduction du facteur correctif $\mathrm{K}$ (cf. IV-B). 
Figure 6 : Modèles de prévision avec et sans ozone du jour comme prédicteur (ChalonChampforgeuil, 30 juillet au 17 août 2003)

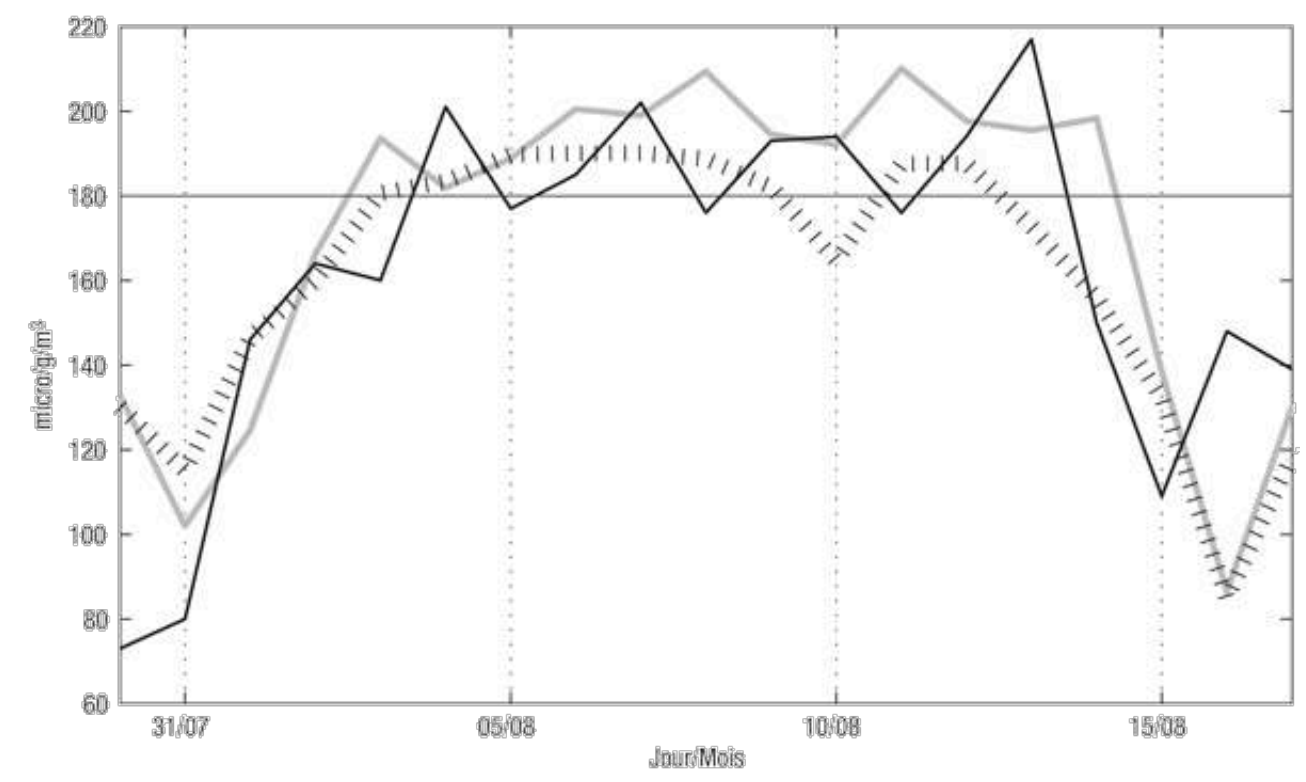

Noir fin: observé ; Grisé épais : prévu ; Barbules : prévu sans ozone du jour.

Pour discriminer le rôle de l'ozone du jour dans les prévisions nous avons refait tourner les modèles en supprimant ce prédicteur et ne conservant que les quatre prédicteurs issus des prévisions météorologiques. Nous sommes donc dans une situation virtuelle d'une atmosphère sans mémoire du point de vue de la chimie de l'ozone. Le déphasage a disparu. Il est donc imputable à la trop grande inertie liée à l'ozone du jour. En revanche, ce modèle se caractérise par une perte de variance expliquée $: R^{2}$ chute de 0.62 à 0.51 pour Chalon-Champforgeuil, et une diminution comparable est observée pour les autres stations. Il s'avère également incapable de prévoir des valeurs réalistes, comme en témoignent les sous-estimations systématiques associées aux épisodes d'ozone où des phénomènes d'accumulation se développent, en particulier en fin d'épisode. Supprimer le prédicteur "ozone du jour ", se traduit ainsi par une dégradation de la prévision les 31 juillet, 7, 9, 10, 12 et 13 août (Figure 6). Il est donc indispensable de considérer les concentrations en ozone du jour pour prévoir celles du lendemain. Ceci est cohérent compte tenu de la chimie de l'ozone, qui, du fait de la complexité des réactions peut se former pendant plusieurs jours à la suite d'émissions de précurseurs (Derwent et al., 2005).

Une autre erreur systématique peut être liée aux différences de concentrations, en précurseurs de l'ozone et en ozone, observée entre les jours ouvrés et les week-ends et jours fériés. Ces différences se traduisent généralement par des concentrations en ozone anormalement élevées dans les villes les week-ends (Jimenez et al., 2005). Ce phénomène est observé en Bourgogne (Houzé, 2000) mais n'est pas suffisamment fort pour que cela soit judicieux de l'intégrer dans les modèles.

54 Les autres erreurs de prévisions ont un caractère non systématique. Il s'agit d'erreurs isolées (dans le temps) mais pouvant être importantes (les plus fortes atteignent $60 \mu \mathrm{g} /$ $\mathrm{m}^{3}$ en positif comme en négatif). De juillet à septembre, des erreurs de prévisions importantes, à savoir supérieures à $|30| \mu \mathrm{g} / \mathrm{m} 3$, interviennent lors de 18 journées sur 92 (soit $20 \%$ environ). Il s'agit de 12 sous-estimations (12, 16, 23, 29 juillet, 2, 7, 10, 13, 19, 27 août, 13, 21 septembre) et de 6 surestimations (2, 24 juillet, 15, 20 août, 3, 5 
septembre). Nous en analysons une de chaque: la surestimation pour le jeudi 14 août et la sous-estimation pour le samedi 16 août. Lors de ces deux journées, les modèles statistico-dynamiques donnent de piètres résultats dans la plupart des 12 stations. Ceux de Chalon-Champforgeuil (tableau 1) et de Dijon-Balzac (tableau 2) révèlent l'ampleur des erreurs (de l'ordre de $50 \mu \mathrm{g} / \mathrm{m}^{3}$ ). Pour tenter d'expliquer ces erreurs, il est logique de se pencher sur les paramètres météorologiques prévus en amont et ;nous confrontons les observations météorologiques effectuées dans les stations MétéoFrance de Chalon-Champforgeuil et de Dijon-Longvic d'une part, avec les prévisions ALADIN sur les points de grilles correspondants.

Tableau 1 : Chalon-sur-Saône : observations et prévisions pour les 14 et 16 août 2003

\begin{tabular}{|c|c|c|c|c|c|c|c|c|}
\hline Jour & T’obs. & T'prév. & RG obs. & RG prev. & Hum. Obs. & Hum. Prév. & $O_{3}$ obs. & $O_{3}$ prév. \\
\hline $14 / 08 / 03$ & 26.3 & 33.8 & 669 & 1127 & 49 & 31 & 150 & 198 \\
\hline $16 / 08 / 03$ & 29.5 & 22.5 & 1016 & 447 & 40 & 82 & 148 & 86 \\
\hline
\end{tabular}

$\mathrm{T}^{\circ}\left({ }^{\circ} \mathrm{C}\right)$ et humidité relative $(\%)$ à $12 \mathrm{~h}$ UTC à Chalon-Champforgeuil. Rayonnement global $\left(\mathrm{W} / \mathrm{m}^{2}\right)$ cumulé depuis le lever du jour à $12 \mathrm{~h}$ UTC à Rully. $\mathrm{T}^{\circ}$, humidité et rayonnement global prévu à $12 \mathrm{~h}$ UTC sur maille ALADIN Chalon-sur-Saône. Ozone $\left(\mu \mathrm{g} / \mathrm{m}^{3}\right)$ prévu et observé à Chalon-Champforgeuil.

Tableau 2 : Dijon : observations et prévisions pour les 14 et 16 août 2003

\begin{tabular}{|c|c|c|c|c|c|c|c|c|}
\hline Jour & T'obs. & Toprév. & $R G$ obs. & $R G$ prev. & Hum. Obs. & Hum. Prév. & $\mathrm{O}_{3}$ obs. & $\mathrm{O}_{3}$ prếv. \\
\hline $14 / 08 / 03$ & 23 & 35.2 & 403 & 1354 & 79 & 29 & 140 & 179 \\
\hline $16 / 08 / 03$ & 28.9 & 25.6 & 1218 & 845 & 48 & 61 & 149 & 97 \\
\hline
\end{tabular}

$\mathrm{T}^{\circ}\left({ }^{\circ} \mathrm{C}\right)$ et humidité relative $(\%)$ à $12 \mathrm{~h}$ UTC à Dijon-Longvic. Rayonnement global $\left(\mathrm{W} / \mathrm{m}^{2}\right)$ cumulé depuis le lever du jour à $12 \mathrm{~h}$ UTC à Dijon-Longvic. $T^{\circ}$, humidité et rayonnement global prévu à $12 \mathrm{~h}$ UTC sur maille ALADIN Dijon. Ozone $\left(\mu \mathrm{g} / \mathrm{m}^{3}\right)$ prévu et observé à Dijon-Longvic.

Les prévisions pour le jeudi 14 août sont largement surestimées. Ceci s'explique 1/ par l'inertie du modèle (à Chalon-Champforgeuil (Figure 6) les concentrations record enregistrées le mercredi 13 août (217 _ g/m3) tirent la prévision vers le haut) et 2/ par l'erreur de prévision météorologique (à Chalon-sur-Saône comme à Dijon le temps est nettement moins chaud et ensoleillé que prévu (tableaux 1 et 2)). Les prévisions pour le samedi 16 août sont, a contrario, largement sous-estimées. La journée est nettement plus chaude et ensoleillée que prévu. L'humidité relative est bien plus faible, à Chalonsur-Saône (tableau 1) comme à Dijon (tableau 2). Notons que ces erreurs météorologiques a priori importantes ne sont pas si rares. Il s'agit dans la plupart des cas d'erreurs associés à un changement de temps qui intervient quelques heures plus tôt (cas de la dégradation du 14 août) ou plus tard (cas de l'amélioration du 16 août) que prévu. Toutefois, cette analyse n'est pas surprenante, car les méthodes statistiques de sortie de modèle dynamique ne peuvent bien sur pas gommer les erreurs ou approximations des prévisions atmosphériques.

\section{Conclusion}

Enjeu important en matière d'effets sur la santé, la prévision des pics de concentrations d'ozone dans l'air ambiant a bénéficié en Bourgogne d'un projet ambitieux de recherche en collaboration entre l'université, Météo-France et ATMOSF'air. Basées sur une méthode d'adaptation statistique de sortie du modèle atmosphérique ALADIN, les 
prévisions de concentrations maximales journalières d'ozone donnent des résultats très encourageants. Compte tenu de la difficulté classique à prévoir des valeurs extrêmes (qui sont en matière de concentrations polluantes les seules réellement intéressantes de manière à favoriser la prévention) et du caractère tout à fait exceptionnel des conditions atmosphériques de la période de validation (printemps-été 2003, considérée actuellement comme la canicule référence), il est rare et donc remarquable, pour un premier essai, d'afficher un taux de réussite de prévisions supérieur à $80 \%$ et une grande robustesse de la méthode. Les résultats obtenus démontrent donc, sans oublier qu'en amont les prévisions météorologiques gardent certaines limites, la faisabilité, en Bourgogne mais sans doute sous d'autres cieux, d'un système opérationnel de prévision des pics de concentration d'ozone. Ceci souligne aussi la nécessité absolue de disposer des modèles dynamiques de prévisions atmosphériques les plus fins tout comme d'excellents réseaux de mesure de la qualité de l'air, données fondamentales sans lesquelles la mise en œuvre de ce type de prévisions resterait à l'état de projet.

\section{BIBLIOGRAPHIE}

Bavay R., Roussel I. (1992). - « Conditions atmosphériques et pollution photo-oxydante en Europe du Nord-Ouest : l'exemple de l'été 1989 ", Pollution Atmosphérique, vol 34, n 135, pp. 36-49.

Derwent R.G., Jenkin M.E. Saunders S.M., Pilling M.J., Passant N.R. (2005). - “Multi-day ozone formation for alkenes and carbonyls investigated with a master chemical mechanism under European conditions". Atmospheric Environment, Vol. 39, pp. 627-635.

Durand P., Cros B. ( 2004). - « Une expérience d'étude de la pollution atmosphérique à l'échelle régionale. Le programme Escompte ». La Météorologie, Vol. 44, pp.16-27.

Fromage A., Gilibert E. (1997). - « Prévision des épisodes d'ozone : état de l'art dans le monde ", Pollution Atmosphérique, Vol. 39, n 154, pp. 52-59.

Ghiaus C.(2005). - "Linear fuzzy-discriminant analysis applied to forecast ozone concentration classes in sea-breeze regime”. Atmospheric Environment, Vol. 39, n 26, pp. 4691-4702.

Grazzini F., Ferranti L., Lalaurette F., Vitart F.(2003). - "The exceptional warm anomalies of summer 2003". ECMWF Newsletter, 99, pp. 2-8.

Houzé M.-L. (1999). - Concentrations en ozone dans les agglomérations dijonnaise et chalonnaise et conditions météorologiques (avril-août 1998), Dijon, université de Bourgogne, 66 p.

Houzé M.-L., Chevrand P., Richard Y. (2003). - « Apports des données ALADIN dans le cadre d'une prévision de l'ozone troposphérique en Bourgogne ", Actes des $6^{\text {èmes }}$ rencontres de Théo Quant, http://thema.univ-fcomte.fr/theoq/pdf/ 2003/03Houze.pdf, 8 p.

Houzé M.-L., Richard Y., Monteiro S. (2000). - « Prévisibilité à 24 h de la pollution en ozone à Dijon et Chalon-sur-Saône ", Pub. Ass. Int. Climatologie, Vol. 13, pp. 500-508. 
Jacob D.J. (2000). - "Heterogeneous chemistry and tropospheric ozone", Atmospheric Environment, Vol. 34, pp. 2131-2159.

Jimenez P., Parra R., Gasso S., Baldasano J.M. (2005). - “Modelling the ozone weekend effect in very complex terrains: a case study in the Northeastern Iberian Peninsula", Atmospheric Environment, Vol. 39, $\mathrm{n}^{\circ}$ 3, pp 429-444.

Luchetta L., Simon V., Torres L. (2000). - « Émission des principaux Composés Organiques Volatils biogéniques en France ", Pollution Atmosphérique, Vol. 42, n¹67, pp. 387-409.

McMillan N., Bortnick S.M., Irwin M.E., Berlinger L.M. (2005). - “A hierarchical Bayesian model to estimate and forecast ozone through space and time”, Atmospheric Environment, Vol. 39, n 8, pp 1373-1382.

Megie G., Bonte J., Carlier P., Chavaudra J., Dizengremel P., Feugier A., Granier C., Hauglustaine D., Kanakidou M., Le Bras G., Marenco A., Mouvier G., Tissot B., Toupance G., Truhaut R., - dir. (1993). - Ozone et propriétés oxydantes de la troposphère, Paris : Lavoisier, Coll. Tech. \& Doc., Rapport $\mathrm{n}^{\circ} 30,253 \mathrm{p}$.

Pailleux J., Geleyn J.-F., Legrand E. (2000). - « La prévision numérique du temps avec les modèles ARPEGE et ALADIN ", La Météorologie, Vol. 30, pp. 32-60.

Richard Y., Houzé M.-L., Chabin J.-P., Thevenin D. (2000). - « Pollution en ozone en Bourgogne orientale ", Pub. Ass. Int. Climatologie, 13, pp. 528-537.

Solberg S., Bergström R., Langner J., Laurila T., Lindskog A. (2005). - “Changes in Nordic surface ozone episodes due to European emission reductions in the 1990s", Atmospheric Environment, Vol. 39, pp. 179-192.

Thiria S., Lechevallier Y., Gascuel O., Canu S. (1997). - Statistiques et méthodes neuronales,Paris, Ed. Dunod, 311 p.

Vautard R., Beekmann M., Roux J., Gombert D. (2000). - "Validation of a hybrid forecasting system for the ozone concentrations over the Paris area", Atmospheric Environment, Vol. 35, pp. 2449-2461.

Vautard R., Honoré C., Beekmann M., Rouil L. (2005). - "Simulation of ozone during the August 2003 heat wave and emission control scenario", Atmospheric Environment, Vol. 39, pp. 2957-2967.

Vergez G. (1999). - Prévisions des pointes d'ozone dans l'agglomération lyonnaise. Note de travail de l'école nationale de la météorologie, $n^{\circ} 669,92 \mathrm{p}$.

Ward M.N., Folland C.K. (1991). - "Prediction of seasonal rainfall in the Nordeste of Brazil using eigenvectors of sea-surface temperature", International Journal of Climatology, Vol. 17, pp. 711-743.

\section{NOTES}

1. Les COVNM (Composés Organiques Volatiles Non Méthaniques) ne sont pas mesurés en Bourgogne. Dans les milieux riches en NOx (> $5 \mathrm{ppb}$ ) la production d'ozone augmente avec celle des COVNM (Luchetta et al. 2000). Le fait que les modèles statistiques établis ne prennent pas en compte les émissions biogéniques ou anthropogéniques de COVNM pourrait expliquer certaines approximations des résultats. 


\section{RÉSUMÉS}

Anticiper de 24 heures les pics de concentration d'ozone atmosphérique par des méthodes simples à mettre en œuvre pouvait paraître très délicat. Cette étude originale, menée en collaboration multidisciplinaire en Bourgogne, prouve la faisabilité de ce type de prévisions par des méthodes fiables et éprouvées, basées sur des adaptations statistiques des sorties du meilleur modèle opérationnel de prévisions météorologiques français ALADIN. S'il est illusoire de faire mieux que la prévision météorologique en amont, les résultats de ces prévisions sont bien plus qu'encourageants, y compris pendant l'exceptionnelle canicule de l'été 2003. Cette étude ouvre donc la voie vers une meilleure anticipation des pics d'ozone, et donc une amélioration de l'information du public, des autorités et du monde médical, en Bourgogne comme ailleurs.

To foresee, one day, the peaks of atmospheric ozone concentration, using simple computational methods, could appear very contentious. This original, collaborative, multidisciplinary study, undertaken in Burgundy, proves the feasibility of this type of forecast, by reliable and tested methods, based on statistical adaptations of the outputs of the best operational model of atmospheric forecasts, the French model ALADIN. Although it may be illusory to do better than weather forecasting, the results of these ozone forecasts, including that during the exceptional heat wave of summer 2003, are much more than just encouraging. This study opens the way towards a better forecast of ozone peaks, and therefore an improvement in information supplied to the public, to authorities and to the medical profession, in Burgundy and elsewhere.

Die Prognose von Spitzenwerten atmosphärischer Ozonkonzentration 24 Stunden im Voraus, erschien bislang recht schwierig. Diese neuartige Studie, durchgeführt von einem interdisziplinären Forscherteam aus dem Burgund, beweist die Machbarkeit dieser Art von Vorhersagen auf der Grundlage bewährter Methoden, die auf den statistischen Anpassungen von Berechnungen des besten einsatzfähigen, französischen Modells für Wettervorhersagen "ALADIN" basieren. Selbst für die außergewöhnliche Hitzewelle des Sommers 2003 waren die Ergebnisse der Prognosen sehr zufriedenstellend. Die Ergebnisse der Studie zeigen damit neue Möglichkeiten für eine verbesserte Vorhersage der maximalen Ozonbelastung auf. Nicht nur im Burgund können deshalb, die Öffentlichkeit, die Behörden und das Gesundheitswesen zukünftig besser informiert werden.

INDEX

Mots-clés : Bourgogne, Ozone, prévision

Schlüsselwörter : Burgund, Ozon, Prognose

Keywords : Burgundy, forecast, Ozone

\section{AUTEURS}

\section{YVES RICHARD}

Centre de Recherches de Climatologie - UMR 5210 CNRS/Université de Bourgogne - 6, bd Gabriel, 21000 Dijon - yves.richard@u-bourgogne.fr 


\section{MARIE-LAURE HOUZE}

Centre de Recherches de Climatologie - UMR 5210 CNRS/Université de Bourgogne - 6, bd Gabriel, 21000 Dijon - marie-laure.houze@u-bourgogne.fr

SERGE TABOULOT

Météo-France Dijon - 22-24 rue Louis de Broglie, 21000 Dijon - serge.taboulot@meteo.fr 